\title{
Produção de cultivares de feijão submetidas a adubação foliar molíbdica ${ }^{1}$
}

\author{
Ricardo Barros Silva ${ }^{2}$, Wemerson Saulo da Silva Barbosa², João Cardoso de Albuquerque Neto ${ }^{3}$,
} Wellington Manoel dos Santos ${ }^{2}$, Antônio Lucrécio dos Santos Neto ${ }^{3}$, Ademária Aparecida de Souza ${ }^{3}$

\begin{abstract}
Resumo: Pesquisas voltadas a seleção de cultivares de feijão e adubação foliar molíbdica no estado de Alagoas ainda são escassas. Neste experimento avaliou-se o desempenho de cultivares de feijão comum sob ausência e presença de adubação foliar molíbdica no Agreste alagoano. O experimento foi conduzido entre maio e julho de 2016 no município de Arapiraca - AL. O delineamento utilizado foi em blocos casualizados, com quatro repetições, em um arranjo 5 x 2, referente as cultivares de feijão (BRS Ametista, BRS Cometa, BRS Estilo, BRS Notável e BRS Pérola) e presença e ausência da adubação foliar molíbdica. O potencial produtivo das cultivares e a interferência da adubação foliar rica em molibdênio foi verificada através de análise estatística das variáveis: produtividade $\left(\mathrm{kg} \mathrm{ha}^{-1}\right)$, número de sementes por planta, número de vagens, número de sementes por vagem e peso de sementes por planta (g). A adubação foliar molíbdica e a interação cultivares $\mathrm{x}$ adubação foliar molíbdica foram não significativas. As cultivares não apresentaram diferença para o número de sementes e vagens por planta. A cultivar BRS Ametista apresentou o maior peso de sementes por planta $(18,17 \mathrm{~g})$, por conseguinte a maior produtividade (3.750 $\left.\mathrm{kg} \mathrm{ha}^{-1}\right)$, seguida da cultivar BRS Estilo $\left(3.126 \mathrm{~kg} \mathrm{ha}^{-1}\right)$, ambos materiais mostraram-se como candidatos promissores sob as condições edafoclimáticas trabalhadas. As Cultivares BRS Ametista e Estilo são as mais produtivas no Agreste alagoano. O molibdênio via foliar aos 35 e 45 dias após a semeadura não é viável.
\end{abstract}

Palavras-chave: adaptabilidade; genótipos; molibdênio; Phaseolus vulgaris L.

\section{Bean cultivars production subjected to molybdenum foliar fertilization}

\begin{abstract}
Research aimed at selection of bean cultivars and molybdenum leaf fertilization in Alagoas State is still scarce. In this experiment, we evaluated the performance of cultivars of common beans in the absence and presence of molybdenum folair fertilization of Alagoas State. The experiment was carried out between May and July 2016 on Arapiraca city - AL. The experimental design was a randomized block, with four replications, in an arrangement $5 \times 2$, concerning the five bean cultivars (BRS Amethyst BRS Comet BRS Style BRS Remarkable and BRS Pearl) and presence and absence of molybdenum leaf fertilization. The yield potential of cultivars and interference of molybdenum rich foliar fertilization was checked by statistical analysis of the variables productivity $\left(\mathrm{kg} \mathrm{ha}^{-1}\right)$, number of seeds per plant, number of pods, number of seeds per pod and seed weight per plant $(\mathrm{g})$. The molybdenum foliar fertilization factor and interaction cultivar $\mathrm{x}$ molybdenum foliar fertilization showed no significance. The cultivate showed no significance for the variables number of seeds and pods per plant. However, there was significance for the other variables, the BRS Ametista showed the highest seed weight per plant (18.17 $\mathrm{g}$ ), thus greater productivity $\left(3,750 \mathrm{~kg} \mathrm{ha}^{-1}\right)$, followed by BRS Estilo $\left(3,126 \mathrm{~kg} \mathrm{ha}^{-1}\right)$, both varieties showed itself as promising candidates under the worked soil and weather conditions. BRS Amethyst and Style cultivars are the most productive in the Alagoas Agreste. Leaf molybdenum at 35 and 45 days after sowing is not viable.
\end{abstract}

Keywords: adaptability; genotypes; molybdenum; Phaseolus vulgaris L.

\footnotetext{
${ }^{1}$ Submetido em 24/08/2018 a aprovado em 19/09/2019;

${ }^{2}$ Programa de Pós-Graduação em Agronomia (Produção Vegetal), Universidade Federal de Alagoas, Rio Largo, Alagoas, Brasil; E-mail: ricardoufa12010@gmail.com - ORCID: 0000-0002-0127-8325; agrowssb@gmail.com - ORCID: 0000-0002-4597-3215; wellington.ea@ hotmail.com - ORCID: 0000-0002-3522-8596;

${ }^{3}$ Programa de Pós-Graduação em Agricultura e Ambiente, Universidade Federal de Alagoas, Arapiraca, Alagoas, Brasil; E-mail: joaocardosoneto@hotmail.com; santosneto@gmail.com - ORCID: 0000-0003-1540-0202; ademariasouza@yahoo.com.br - ORCID: 0000-00031540-0202.
}

Agropecuária Técnica, Areia-PB, v. 40, n. 1-2, p. 14-20, 2019

https://doi.org/10.25066/agrotec.v40i1-2.42376 


\section{Introdução}

O feijão é uma das culturas mais produzidas no mundo, gerando emprego e renda nas regiões produtoras. A cultura apresenta grande importância no combate à desnutrição em muitas regiões do planeta, sendo rica em carboidratos, ferro e vitaminas (Fabbri et al., 2016; Chekanai et al., 2018). A região Agreste do estado de Alagoas detém condições climáticas favoráveis para o cultivo do feijão comum (temperaturas entre $18 \mathrm{e}$ $30{ }^{\circ} \mathrm{C}$, precipitação pluvial durante o ciclo de 300 a $600 \mathrm{~mm}$ ) (Filgueira, 2008) ao contrário de muitas regiões nordestinas. Nimer (1977) caracteriza o clima local com uma precipitação pluvial anual entre 750 e $1000 \mathrm{~mm}$ e apresentando uma boa disponibilidade térmica durante todo o ano.

A utilização de técnicas inadequadas em cultivos, aliada ao baixo índice tecnológico empregado nas diversas regiões brasileiras, além de fatores impactantes no cultivo do feijão, como, períodos de seca prolongados e ataque de pragas e doenças, contribuem de forma preponderante para a baixa produtividade obtida em diversas regiões brasileiras (Melo et al., 2007). Tais fatores podem ter seu impacto reduzido através da adoção de cultivares tolerantes a tais problemas comuns em cultivos (Boydston et al., 2018). Diante do exposto, a seleção de cultivares de feijão aptas a condições edafoclimáticas de uma determinada região surge como fator importante na busca por aumento da produtividade e tolerância a fatores abióticos e bióticos que atuam em áreas de cultivo.

Dentre as técnicas empregadas de forma inadequada no manejo da cultura do feijão, destaca-se a adubação. Verifica-se grande aplicação irracional de fertilizantes em cultivos de feijão, principalmente em áreas geridas por pequenos e médios produtores. Este erro é comum em Alagoas pelo fato de cultivos no estado serem realizados sob baixo índice tecnológico, onde os agricultores não possuem o hábito de realizar análise de solo e não recebem assistência técnica.

Dentre os nutrientes mais exigidos pela cultura do feijão destaca-se o nitrogênio, que é obtido principalmente pela fixação biológica, resultado da simbiose entre o feijoeiro e bactérias diazotróficas habitantes do solo (Pessoa et al., 2000; Rufini et al., 2011). Para que a simbiose ocorra entre o feijoeiro e a bactéria, torna-se importante a suplementação do nutriente molibdênio no cultivo, onde este quase sempre é disponibilizado via foliar.

O molibdênio atua como componente estrutural da nitrogenase e redutase, enzimas que participam do metabolismo do nitrogênio e sua disponibilização para a planta (Epstein, 1975; Oliveira et al., 2017). Logo, a falta deste nutriente afeta de forma direta o metabolismo do nitrogênio, podendo causar queda na produtividade, principalmente em solos pobres nutricionalmente. Em Alagoas a adubação molíbdica em cultivos de feijão não é comum, surgindo como um agravante e favorecendo a baixa produtividade do estado, $531 \mathrm{~kg} \mathrm{ha}^{-1}$ (IBGE, 2018).

Dado o exposto, observa-se que trabalhos destinados a seleção de cultivares aliados a tratos culturais adequados no manejo da cultura apresentam grande importância, podendo resultar no aumento da produtividade em cultivos no Agreste alagoano. Assim, o objetivo com esta pesquisa foi avaliar o desempenho de cultivares de feijão comum e da adubação foliar molíbdica sobre características agronômicas.

\section{Material e Métodos}

\subsection{Descrição da área experimental}

O experimento foi conduzido na área experimental do Campus Arapiraca da Universidade Federal de Alagoas, na cidade de Arapiraca $\left(9^{\circ} 45^{\prime} 58^{\prime \prime}\right.$ de latitude Sul e $35^{\circ} 38^{\prime} 58^{\prime \prime}$ de longitude Oeste, $264 \mathrm{~m}$ de altitude), Agreste de Alagoas. O período do cultivo foi de 13 de maio a 30 de julho de 2016. Nimer (1977) caracteriza o clima da região com temperatura média anual de $25^{\circ} \mathrm{C}$, precipitação pluvial anual média entre 750 e $1000 \mathrm{~mm}$, onde $50 \%$ da chuva anual concentrase nos meses de maio, junho e julho, sendo a primavera e o verão o período seco da região.

$\mathrm{O}$ solo da área experimental foi classificado como um Latossolo Vermelho distrófico (Embrapa, 2013), onde antes da implantação do experimento realizou-se a coleta de amostras na camada de 0 a $20 \mathrm{~cm}$ para análise (Tabela 1). O preparo do solo foi realizado através de duas gradagens leves, sendo a primeira 30 e a segunda 5 dias antes da semeadura. 
Tabela 1 Características químicas do solo da área experimental

\begin{tabular}{|c|c|c|c|c|c|c|c|c|c|c|}
\hline $\mathrm{pH}$ & $\mathrm{K}^{+}$ & $\mathrm{P}$ & $\mathrm{Na}^{+}$ & $\mathrm{Ca}^{2+}$ & $\mathrm{Mg}^{2+}$ & $\mathrm{Al}^{3+}$ & $\mathrm{H}+\mathrm{Al}$ & CTC & SB & V \\
\hline & $\mathrm{mg} \mathrm{d}$ & & & & $-\mathrm{cmol}_{\mathrm{c}}$ & $\mathrm{n}^{-3}$----- & & & \multicolumn{2}{|c|}{$\%$} \\
\hline 5,6 & 109 & 11 & 12 & 2,1 & 0,8 & 0,09 & 1,2 & 2,62 & 2,53 & 67,8 \\
\hline
\end{tabular}

\subsection{Delineamento e tratamentos}

O experimento ocupou uma área total de 112,5 $\mathrm{m}^{2}$. Adotou-se o delineamento em blocos casualizados, com os tratamentos em esquema fatorial 5 × 2, sendo as cultivares de feijão (BRS Ametista, BRS Estilo, BRS Cometa, BRS Notável e BRS Pérola) e as adubações foliares molíbdica (presença e ausência), com quatro repetições, totalizando 40 parcelas experimentais. As parcelas possuíram as dimensões de 1,5 x 1,5 $\mathrm{m}$, onde cada parcela foi constituída por 3 linhas de cultivo espaçadas por $0,5 \mathrm{~m}$. Considerou-se como área útil a porção central da parcela $(0,5$ $\mathrm{m}^{2}$ ), desprezando as linhas marginais como bordadura. A semeadura foi realizada manualmente em uma densidade de 0,08 $\mathrm{m}$ entre plantas, correspondendo 12 sementes por metro linear.

\subsection{Teste de germinação}

Previamente realizou-se um teste de germinação de acordo com as Regras para Análise de Sementes (Brasil, 2009), objetivando verificar o potencial germinativo dos lotes de sementes (Tabela 2).

Tabela 2 Teste de germinação para verificação da qualidade dos lotes de sementes de feijão comum

\begin{tabular}{lc}
\hline \multicolumn{1}{c}{ Cultivares } & Germinação (\%) \\
\hline Ametista & 100 \\
Cometa & 99 \\
Estilo & 99 \\
Notável & 92 \\
Pérola & 99 \\
\hline
\end{tabular}

Mediante os resultados obtidos, verificou-se uma porcentagem de germinação satisfatória para todas as cultivares, garantindo um estande homogêneo no campo.

\subsection{Tratos culturais}

A adubação de fundação e de cobertura foi realizada conforme a recomendação descrita por Cavalcanti (2008). Na adubação de fundação utilizou-se o formulado 04-14-08 como fonte de fósforo, necessitou-se $105 \mathrm{~kg}^{-1}$ de $\mathrm{P}_{2} \mathrm{O}_{5}$, resultando em uma distribuição de $37,5 \mathrm{~g} \mathrm{~m}^{-1}$ do formulado. A adubação de cobertura foi realizada 13 dias após a emergência da cultura, utilizou ureia como fonte de nitrogênio, necessitou-se 60 $\mathrm{kg} \mathrm{ha}^{-1}$ de N, distribuindo $6,6 \mathrm{~g} \mathrm{~m}^{-1}$ do fertilizante nitrogenado. Durante o ciclo da cultura também foi realizado tratos culturais, como, capinas e controle de pragas. As capinas foram realizadas manualmente com auxílio de enxadas. Para o controle de pragas utilizou-se o método químico, com uso de inseticida piretróide.

\subsection{Adubação foliar molíbdica}

A aplicação do tratamento adubação foliar molíbdica foi realizada aos 35 e 45 dias após a semeadura, período caracterizado pelo início do florescimento e surgimento das primeiras vagens. Utilizou-se como fonte de molibdênio o produto comercial Potamol $^{\circledR}$, sendo composto por $14,0 \%$ de molibdênio e $12,0 \%$ de potássio. Utilizou-se a dosagem de $0,5 \mathrm{~L}$ do produto em $100 \mathrm{~L}$ de água. Aplicou-se o produto com pulverizador costal com capacidade de 5 L, bico TEEJET 8002, DMV médio, pressão de cerca de $180 \mathrm{Ibf} / \mathrm{pol}^{2}$. O horário de aplicação foi 7:00 às 9:00 horas da manhã, em dias não nublados, sob uma temperatura de $26{ }^{\circ} \mathrm{C}$ e umidade relativa do ar de $72 \%$. Uma lona plástica foi utilizada objetivando evitar o efeito da deriva entre as parcelas experimentais.

\subsection{Avaliações e colheita}

A colheita do feijão foi realizada 73 dias após a semeadura quando as plantas encontravam-se em senescência. Avaliou-se no campo durante a colheita a variável número de vagens por planta. Posteriormente as vagens foram levadas para um laboratório e obteve-se as variáveis: número de sementes por vagem, número de sementes por planta, peso de sementes por planta $(\mathrm{g})$ e produtividade $\left(\mathrm{kg} \mathrm{ha-}{ }^{-1}\right)$. Para a obtenção da produtividade corrigiu-se a umidade para $13 \%$ (base úmida), determinada por meio do método de estufa a $105^{\circ} \mathrm{C}$ por $24 \mathrm{~h}$ (BRASIL, 2009). A 
obtenção do peso de sementes por planta foi realizada através de balança com precisão $0,001 \mathrm{~g}$.

\subsection{Análise estatística}

Os dados obtidos foram submetidos a análise de variância utilizando o software estatístico SISVAR (Ferreira, 2011), os tratamentos que mostraram-se significativos pelo teste $\mathrm{F}$, tiveram suas médias comparadas pelo teste de Scott-Knott a $5 \%$ de significância.

\section{Resultados e Discussões}

Os efeitos das cultivares e da adubação foliar com molibdênio nas características produtivas dos feijoeiros podem ser observados na Tabela 3 . Observou-se diferença significativa apenas em função do fator cultivares para a produtividade, número de sementes por vagem e peso de sementes por planta.

Tabela 3 Resumo das análises de variância (Quadrado Médio) para as variáveis, número de vagens por planta (NVP), produtividade (PROD), número de sementes por vagem (NSV), peso de sementes por planta (PSP) e número de sementes por planta (NSP), em cultivares de feijão-comum em função de adubação foliar molíbdica

\begin{tabular}{ccccccc}
\hline & GL & NSV & PSP & PROD & NVP & NSP \\
\hline Cultivar (C) & 4 & $1,846706^{*}$ & $89,570947^{*}$ & $3232252,51^{*}$ & $12,8214^{\text {ns }}$ & $146,07815^{\text {ns }}$ \\
Ad. Foliar (AF) & 1 & $0,018062^{\text {ns }}$ & $14,042250^{\text {ns }}$ & $3941,21^{\text {ns }}$ & $4,4023^{\text {ns }}$ & $83,405440^{\text {ns }}$ \\
C x AF & 4 & $0,167481^{\text {ns }}$ & $14,216387^{\text {ns }}$ & $195068,12^{\text {ns }}$ & $5,1285^{\text {ns }}$ & $97,362015^{\text {ns }}$ \\
Blocos & 3 & $0,716962^{\text {ns }}$ & $30,764330^{\text {ns }}$ & $835661,34^{\text {ns }}$ & $5,7053^{\text {ns }}$ & $193,66247^{\text {ns }}$ \\
Erro & 27 & 0,633827 & 10,830793 & 271618,65 & 3,6751 & 110,581777 \\
\hline CV $(\%)$ & & 18,38 & 23,57 & 18,48 & 19,93 & 25,55 \\
\hline
\end{tabular}

${ }^{\mathrm{ns}}$ não significativo e *significativo a $5 \%$ de probabilidade, pelo teste $\mathrm{F}(\mathrm{p}<0,05)$.

As cultivares Pérola e Cometa não diferiram entre si para o número de sementes por vargem com média de 4,85 (Figura 1), sendo superiores as Ametista, Estilo e Notável com média de 3,99, redução média de $18 \%$.

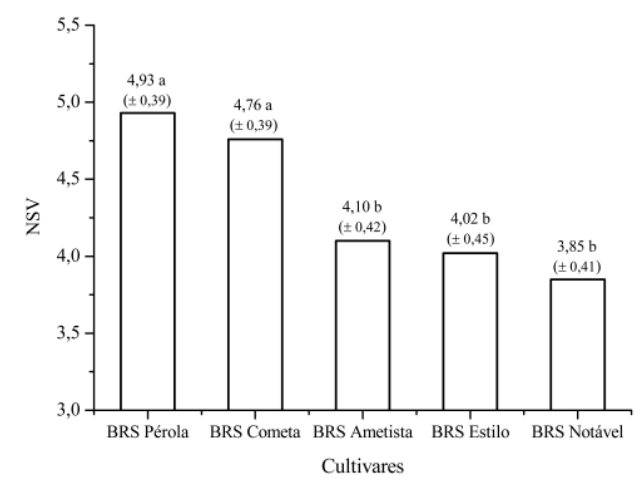

Figura 1 Número de sementes por vagem (NSV) das cultivares estudadas. Médias seguidas da mesma letra não diferem entre si pelo teste de Scott-Knott ( $\mathrm{p}<$ $0,05)$.

O resultado para o número de sementes por vagem corrobora com os obtidos por Nascimento et al. (2009), Biscaro et al. (2009) e Rocha et al. (2011). Estes autores afirmam que o número de sementes por vagem é uma característica de herdabilidade genética, com pouca influência do ambiente e condições de cultivo. Logo, a adubação foliar molíbdica tende a não influenciar neste componente de produção.

$\mathrm{O}$ peso de sementes por planta para as cultivares Ametista, Pérola e Estilo não diferiram entre si (Figura 2) com média de 15,56 g, superando em $47 \%$ a média das cultivares Notável e Cometa que foi de 10,76 g (Figura 2).

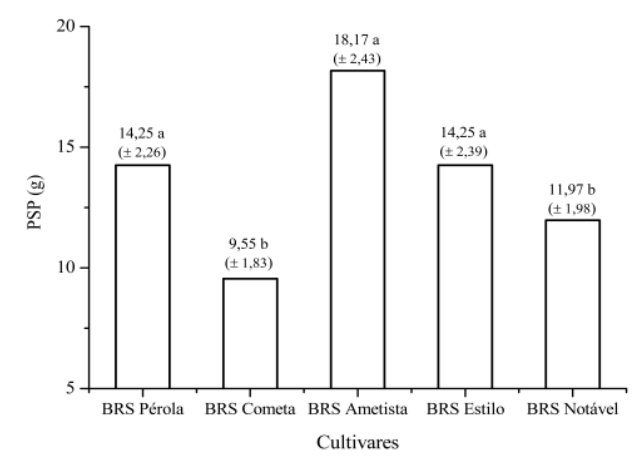

Figura 2 Peso de sementes por planta (PSP) (g) das cultivares estudadas. Médias seguidas da mesma letra não diferem entre si pelo teste de Scott-Knott ( $\mathrm{p}<$ $0,05)$. 
Ascoli et al. (2008) e Rocha et al. (2011) estudando o efeito do molibdênio via foliar sobre o peso de sementes de feijão verificaram resultados semelhantes. Para os autores, a influência do molibdênio via foliar sobre o feijão depende da dose aplicada e condições nutricionais da cultura no momento da aplicação.

A maior produtividade foi registrada para a cultivar Ametista, 3,75 $\mathrm{t} \mathrm{ha}^{-1}$, superando em 20\% a cultivar Estilo com 3,126 t ha $\mathrm{ha}^{-1}$, em $56 \%$ a média das cultivares Pérola, Notável e Cometa que foi de 2,407 $\mathrm{tha}^{-1}$ (Figura 3). A cultivar Estilo supero em $30 \%$ a médias das cultivares Pérola, Notável e Cometa.

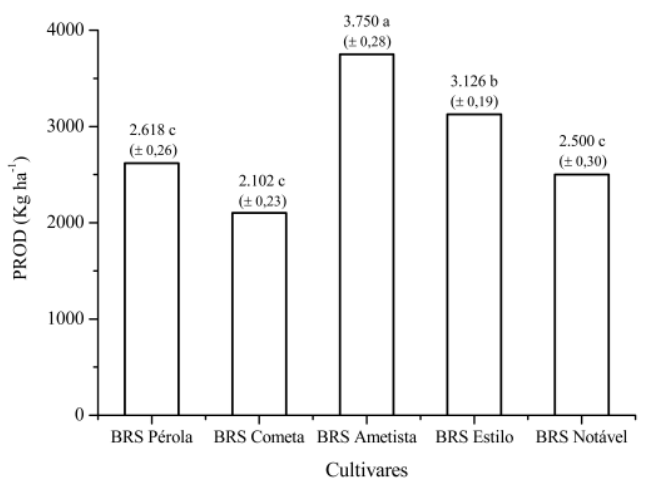

Figura 3 Valores médios de produtividade (PROD) $\left(\mathrm{kg} \mathrm{ha}^{-1}\right)$ das cultivares estudadas. Médias seguidas da mesma letra não diferem entre si pelo teste de ScottKnott $(\mathrm{p}<0,05)$.

O desempenho satisfatório da produtividade da cultivar BRS Ametista em relação a outras cultivares tradicionais no Brasil é confirmado por Melo et al. (2012). De modo geral, as cultivares apresentaram comportamento diversificado em relação as variáveis estudadas. Este comportamento pode estar ligado a interação genótipo ambiente, onde cada cultivar tende a apresentar desempenho específico para as condições edafoclimáticos de cada ambiente de cultivo. Alguns experimentos (Ribeiro et al., 2009; Fernandes et al., 2015) evidenciam a variabilidade que as cultivares podem apresentar em relação a características agronômicas em função das condições de cultivo.

A adubação foliar tem sua eficiência afetada, principalmente, em função das condições climáticas no momento da aplicação como, por exemplo, temperatura, radiação solar e precipitação pluvial (Batista et al., 2017), podendo reduzir a eficiência do fertilizante aplicado. Outros fatores importantes são a época de aplicação do fertilizante foliar em relação ao desenvolvimento fenológico e o grau de nutrição da planta. Estes dois últimos fatores podem ter influenciado preponderantemente nos resultados da pesquisa, evidenciando a importância de mais trabalhos com adubação foliar molíbdica na cultura do feijão no Agreste de Alagoas, testando épocas de aplicação da adubação foliar molíbdica em relação ao desenvolvimento fenológico da cultura. Outras pesquisas também evidenciam que nem sempre a adubação foliar molíbdica propicia ganho produtivo em espécies leguminosas, como o feijoeiro (Possenti et al., 2010; Lopes et al., 2014). Salienta-se que a adubação nitrogenada em grandes quantidades pode diminuir a eficiência simbiótica, mas pequenas quantidades tendem a aumentar o crescimento dos nódulos e elevar a FBN. O baixo teor de nitrogênio no solo também pode ser limitante à atividade simbiótica (HUNGRIA et al., 2003).

As cultivares Ametista e Estilo podem ser consideradas promissoras para cultivos no Agreste alagoano, pois apresentaram desempenho satisfatório em relação as variáveis peso de sementes por plantas e produtividade, características importantes em áreas de cultivo. Todavia, não pode-se confirmar a inviabilidade das demais cultivares para região, tendo em vista que seria necessário a repetição do experimento em anos seguintes e diferentes épocas de semeadura objetivando verificar o comportamento destes genótipos sob diferentes condições climáticas na região.

\section{Conclusões}

As cultivares BRS Ametista e Estilo são as mais produtivas no Agreste de Alagoas;

A aplicação de molibdênio via foliar aos 35 e 45 dias após a semeadura não é viável para a cultura do feijoeiro, pois não houve retorno produtivo.

\section{Referências}

Ascoli, A. A.; Soratto, R. P.; Maruyama, W. I. Aplicação foliar de molibdênio, produtividade e qualidade fisiológica de sementes de feijoeiro irrigado. Bragantia, v. 67 , n. 2 , p. $377-384$, 2008. http://dx.doi.org/10.1590/S0006- 
Batista, V. V.; Adami, P. F.; Giaretta, R.; Rabelo, P. R.; Rosa, L.C. Eficiência de diferentes fertilizantes foliares em três cultivares de soja. Revista Técnico-Científica do CREA-PR, v. 9, p. 1-11, 2017. http://creaprw16.creapr.org.br/revista/Sistema/index.php/revista/article/view/2 35

Biscaro, G. A; Goulart Júnior, S. A. R; Soratto, R. P.; Freitas Júnior, N. A.; Motomiya, A. V. A.; Calado Filho, G. C. Molibdênio via semente e nitrogênio em cobertura no feijoeiro irrigado em solo de cerrado. Ciência e Agrotecnologia, v. 33, n. 5, p. 1280-1287, 2009. http://dx.doi.org/10.1590/S1413-70542009000500012

Boydston, R. A.; Porter, L. D.; Chaves-Cordoba, B.; Khot, L. R.; Miklas, P. N. The impact of tillage on pinto bean cultivar response to drought induced by deficit irrigation. Soil and Tillage Research, v. 180, p. 63-72, 2018. https://doi.org/10.1016/j.still.2018.02.011

BRASIL. Ministério da Agricultura, Pecuária e Abastecimento. Regras para análise de sementes. Ministério da Agricultura, Pecuária e Abastecimento. Secretaria de Defesa Agropecuária. Brasília, DF: MAPA/ ACS, 2009. 395p.

Cavalcanti, F. J. A. Recomendações de adubação para o estado de Pernambuco ( $2^{\mathrm{a}}$ Aproximação). Recife: IPA, 2008. 212 p.

Chekanai, V.; Chikowo, R.; Vanlauwe, B. Response of common bean (Phaseolus vulgaris L.) to nitrogen, phosphorus and rhizobia inoculation across variable soils in Zimbabwe. Agriculture, Ecosystems \& Environment, v. 266, p. 167-173, 2018. https://doi.org/10.1016/j.agee.2018.08.010

EMPRESA BRASILEIRA DE PESQUISA AGROPECUÁRIA - EMBRAPA. Sistema brasileiro de classificação de solos. 3 ed. Brasília, 2013. 353p

EPSTEIN, E. Nutrição mineral das plantas: princípios e perspectivas. Rio de Janeiro: Livros Técnicos e Científicos, 1975.

FABBRI, A. D. T.; SCHACHT, R. W.; CROSBY, G. A. Evaluation of resistant starch content of cooked black beans, pinto beans, and chickpeas. NFS Journal, v. 3, p. 8-12, 2016. https://doi.org/10.1016/j.nfs.2016.02.002

Fernandes, R. C.; Guerra, J. G. M.; Araújo, A. P. Desempenho de cultivares de feijoeiro-comum em sistema orgânico de produção. Pesquisa Agropecuária Brasileira, v. 50, n. 9, p. 797806, 2015. http://dx.doi.org/10.1590/S0100204X2015000900008

FERREIRA, D. F. Sisvar: a computer statical analysis system. Ciência e Agrotecnologia, v. 35, n. 6, p. 1039-1042, 2011. http://dx.doi.org/10.1590/S1413-70542011000600001

Filgueira, F. A R. Novo Manual de Olericultura: agrotecnologia moderna na produção e comercialização de hortaliças. Viçosa: UFV, 2008. 421p.

IBGE - Instituto Brasileiro de Geografia e Estatística. LSPA-Levantamento Sistemático da Produção Agrícola. 2018. https://sidra.ibge.gov.br/home/lspa

Hungria, M.; Campo, R. J.; Mendes, I. C. Benefits of inoculation of the common bean (Phaseolus vulgaris) crop with efficient and competitive Rhizobium tropici strains. Biology and Fertility of Soils, v. 39, n. 2, p. 88-93, 2003. https://doi.org/10.1007/s00374-003-0682-6

Lopes, J. F.; Coelho, F. C.; Rangel, O. J. P.; Rabello, W. S.; Gravina, G. A.; Vieira, H. D. Adubação foliar com níquel e molibdênio no feijoeiro comum cv. Ouro Vermelho. Revista Ceres, v. 61, n. 2, p. 234-240, 2014. http://dx.doi.org/10.1590/S0034-737X2014000200011

Melo, L. C.; Melo, P. G. S.; Faria, L. C.; Diaz, J. L. C.; Del Peloso, M. J.; Rava, C. A.; Costa, J. G. C. Interação com ambientes e estabilidade de genótipos de feijoeiro- comum na Região Centro-Sul do Brasil. Pesquisa Agropecuária Brasileira, Brasília, v. 42, n. 5, p .715-723, $2007 . \quad$ http://dx.doi.org/10.1590/S0100204X2007000500015

Melo, L. C.; Costa, J. G. Costa; Pereira, H. S.; Peloso, M. J. Del; Wendland, A.; Faria, L. C. de; Nascente, A. S.; Díaz, J. L. C.; Carvalho, H. W. L. de; Costa, A. F. da; Almeida, V. M. de; Melo, C. L. P.; Abreu, Â. de F. B.; Magaldi, M. C. de S.; Posse, S. C. P.; Douza Filho, B. F. de; Souza, J. F.; Guimarães, C. M.; Oliveira, J. P. de. Common bean cultivar BRS Ametista with large Carioca grains and disease resistance. Crop Breeding and Applied Biotechnology, v. 12, n. $12, \quad$ p. 293-296, 2012. http://dx.doi.org/10.1590/S1984-70332012000400011

Nascimento, M. S.; Barbosa, G. F.; Buzetti, S.; Nascimento, R. S.; Castro, R. M. Nitrogênio em cobertura e molibdênio via foliar em feijoeiro de 
inverno no sistema plantio direto. Scientia Agraria, v. 10, n. 5, p. 351-358, 2009. http://dx.doi.org/10.5380/rsa.v10i5.15187

\section{Nimer, E. Geografia do Brasil: Região} Nordeste. Rio de Janeiro: IBGE, vol. 2, p. 4784, 1977.

Oliveira, C. A. B.; Pelá, G. M.; Pelá, A. Inoculação com Rhizobium tropici e adubação foliar com Molibdênio na cultura do feijão comum. Revista de Agricultura Neotropical, v. 4, Suplemento 1, p. 43-50, dez. 2017. https://doi.org/10.32404/rean.v4i5.2193

Pessoa, A. C. S.; Ribeiro, A. C.; Chagas, J. M.; Cassini, S. T. A. Concentração foliar de molibdênio e exportação de nutrientes pelo feijoeiro "Ouro Negro" em resposta à adubação foliar com molibdênio. Revista Brasileira de Ciência do Solo, v. 24, n. 1, p. 75-84, 2000. http://dx.doi.org/10.1590/S0100-06832000000100010

Possenti, J. C.; Villela, F. A. Efeito do molibdênio aplicado via foliar e via semente sobre o potencial fisiológico e produtivo de sementes de soja. Revista Brasileira de Sementes, v. 32, n.
4, p. 143-150, 2010. http://dx.doi.org/10.1590/S010131222010000400016

Ribeiro, N. D.; Souza, J. F.; Antunes, I. F.; Poersch, N. L. Estabilidade de produção de cultivares de feijão de diferentes grupos comerciais no estado do Rio Grande do Sul. Bragantia, v. 68, n. 2, p. 339-346, 2009. Http://Dx.Doi.Org/10.1590/S0006-87052009000200007

Rocha, P. R. R; Araújo, G. A. A; Carneiro, J. E. S; Cecon, P. R. Adubação molíbdica na cultura do feijão nos sistemas de plantio de plantio direto e convencional. Revista Caatinga, v. 24, n. 2, p. 9-17, 2011. https://periodicos.ufersa.edu.br/index.php/caati nga/article/view/1926/4706

Rufini, M. Ferreira, P. A. A.; Soares, B. L. Oliveira, D. P. Andrade, M. J. B. Moreira, F. M. S. Simbiose de bactérias fixadoras de nitrogênio com feijoeiro-comum em diferentes valores de pH. Pesq. Agropec. Bras., Brasília, v. 46, n. 1, p. 81-88, 2011. http://www.scielo.br/pdf/pab/v46n1/11.pdf 